\title{
Beneficial effects of a mouthwash containing an antiviral phthalocyanine derivative on the length of hospital stay for COVID-19: Randomised trial
}

Paulo Sérgio da Silva Santos ( $\sim$ paulosss@fob.usp.br)

Bauru School of Dentistry of University of São Paulo

Bernardo Orcina

Bauru School of Dentistry of University of São Paulo

Rafael Machado

Universidade de São Paulo

Fabiano Vilhena

TRIALS - Oral Health \& Technologies

Lucas Alves

Hospital Estadual de Bauru

Mariana Zangrando

Bauru School of Dentistry of University of São Paulo

Rodrigo de Oliveira

Bauru School of Dentistry of University of São Paulo

Mariana Soares

Faculdade São Leopoldo Mandic

Andréa Simão

Londrina State University

Emilene Pietro

Hospital Estadual de Bauru

Juliana Kuroda

Hospital Estadual de Bauru

Ivanilda Benjamim

Hospital Estadual de Bauru

Danielle Araújo

Universidade de São Paulo

Sergio Toma

Universidade de São Paulo

Lourival Flor

Golden Corporation Techonogy

Koiti Araki 
Universidade de São Paulo

\section{Edison Durigon}

Universidade de São Paulo

\section{Research Article}

Keywords: Coronavirus Infections, In Vitro Techniques, Antiviral Agents, Mouthwashes, Length of Stay

Posted Date: April 13th, 2021

DOI: https://doi.org/10.21203/rs.3.rs-365425/v1

License: (1) This work is licensed under a Creative Commons Attribution 4.0 International License. Read Full License 


\section{Abstract}

Background: The risk of contamination and dissemination by SARS-CoV-2 has a strong link with nasal, oral and pharyngeal cavities. Recently, our research group observed the promising performance of an anionic phthalocyanine derivative (APD) used in a mouthwash protocol without photoexcitation; this protocol improved the general clinical condition of patients infected with SARS-CoV-2.

Methods: The present two-arm study evaluated in vitro the antiviral activity and cytotoxicity of APD. Additionally, a triple-blind randomized controlled trial was conducted with 41 hospitalized patients who tested positive for COVID-19. All the included patients received World Health Organization standard care hospital treatment (non-intensive care) plus active mouthwash (experimental group $A M / n=20$ ) or nonactive mouthwash (control group NAM/n=21). The adjunct mouthwash intervention protocol used in both groups consisted one-minute gargling/rinsing / 5 times/day until hospital discharge. Groups were compared considering age, number of comorbidities, duration of symptoms prior admission and length of hospital stay (LOS). The associations between group and sex, age range, presence of comorbidities, admission to Intensive care unit (ICU) and death were also evaluated.

Results: The in vitro evaluation demonstrated that APD compound was highly effective for reduction of SARS-CoV-2 viral load in the $1.0 \mathrm{mg} / \mathrm{mL}$ (99.96\%) to $0.125 \mathrm{mg} / \mathrm{mL}$ (92.65\%) range without causing cytotoxicity. Regarding the clinical trial, the median LOS of the AM group was significantly shortened (4 days) compared with that of the NAM group (7 days) $(p=0.0314)$. Additionally, gargling/rinsing with APD was very helpful in reducing the severity of symptoms (no ICU care was needed) compared to not gargling/rinsing with APD (28.6\% of the patients in the NAM group needed ICU care, and $50 \%$ of this ICU subgroup passed way, $\mathrm{p}=0.0207)$.

Conclusions: This study indicated that the mechanical action of the protocol involving mouthwash containing a compound with antiviral effects against SARS-CoV-2 may reduce the symptoms of the patients and the spread of infection. The use of APD in a mouthwash as an adjuvant the hospital COVID19 treatment presented no contraindication and reduced the hospital stay period.

Trial Registration: The clinical study was registered at REBEC - Brazilian Clinical Trial Register (RBR$58 \mathrm{ftdj}$ ) in 10/28/2020.

\section{Background}

SARS-CoV-2 was first recognized at the end of 2019 , and its outbreak caused a global pandemic that is affecting people all over the world due to it higher contamination rate, spreading capacity and index of lethality than those of previous coronaviruses [1,2,3]. Patients who test positive for COVID-19 are admitted to hospitals and receive intensive care at unprecedent rate [4]. The patients who are most vulnerable to the development of COVID-19 are elderly people who suffer from comorbidities, such as high blood pressure, obesity, heart diseases, breathing problems and neoplasia [4]. Thus, according to the 
World Health Organization (WHO), an early and accurate diagnosis is crucial to control the spread of SARS-CoV-2 because a higher viral load is related to more severe disease $[5,6]$.

It is clear that washing hands, wearing masks, and social distancing are effective measures to fight the pandemic [6, 7]. In addition, considering that COVID-19 contagion, evolution and dissemination have strong associations with the mouth [8,9], gargling with antiseptic mouthwashes has been suggested as an extra preventive measure against COVID-19 [7, 8, 10,11, 12,13,14,15,16,17]. However, in the cases of diseases, such as influenza, which are caused by SARS-type viruses, virucidal activity is essential for such measures to be effective [8].

The potential of phthalocyanines for biological and medical applications has been recognized $[18,19,20$, 21], especially in photodynamic therapy, since phthalocyanines in the excited state can promote the reactive oxygen species generation or redox processes, while no such properties are observed in the absence of light. Recently, our research group observed the promising performance of an anionic phthalocyanine derivative (APD) used in a mouthwash protocol without photoexcitation; this protocol improved the general clinical condition of patients with SARS-CoV-2 infection [14,15].

Hence, the following study aimed to (1) evaluate the antiviral activity and cytotoxicity of APD in vitro and (2) clinically assess the use of an APD-containing mouthwash in hospitalized patients who tested positive for COVID-19 to reduce the severity of the disease and minimize the LOS.

\section{Methods}

This two-arm study consisted of laboratory experiments to evaluate the antiviral activity and cytotoxicity of the anionic iron tetracarboxyphthalocyanine derivative (APD) (Golden Technology Corp., Brazil) and a triple-blind randomized controlled trial.

\section{Laboratory studies}

All the in vitro experiments were conducted in Biosafety Level (BSL) BSL-2 and BSL-3 facilities at the Institute of Biomedical Sciences, University of São Paulo, Brazil, according to the laboratory biosafety guidance recommended by the WHO for the novel coronavirus (SARS-CoV-2) [4].

\section{- Antiviral and cytotoxic activity of APD}

To determine the antiviral activity and cytotoxicity of APD, a $2.0 \mathrm{mg} / \mathrm{mL}$ (I) stock solution prepared in sterile distilled water was serially diluted by 2 -fold $\left(1.0 \mathrm{mg} / \mathrm{mL}\right.$ to $0.39 \times 10^{-2} \mathrm{mg} / \mathrm{mL}$, i.e., $1 / 2$ to $\left.1 / 512\right)$ in Dulbecco's modified essential medium (DMEM) supplemented with $2.5 \%$ fetal bovine serum (FBS) in a 96-well cell culture plate to a final volume of $100 \mu \mathrm{L}$ per well. The dilutions were made in quadruplicate to determine both virus neutralization and cytotoxicity. 
After dilution, $100 \mu \mathrm{L}$ of SARS-CoV-2 (SARS.CoV2/SP02.2020. HIAE. Br) at $10^{3} \mathrm{TCID}_{50} / \mathrm{mL}(\mathrm{MOI}=0,02)$ was added to the wells and incubated for $30 \mathrm{~min}$ at $37^{\circ} \mathrm{C}$. Then, $150 \mu \mathrm{L}$ of the mixture (APD plus virus) was transferred to a 96 -well cell culture plate previously seeded with $1 \times 10^{5}$ Vero CCL-81 cells $/ \mathrm{mL}$ and grown to $80-90 \%$ confluence. The cells were then incubated at $37^{\circ} \mathrm{C}$ in a $5 \% \mathrm{CO}_{2}$ atmosphere for $72 \mathrm{~h}$ [22].

The plate was visually evaluated using an optical microscope to determine cell integrity and morphology, and then, samples were collected (in quadruplicate) for RNA extraction and real-time Polymerase Chain Reaction (RT-PCR) for the quantitative detection of the amount of active virus. The cells were fixed with Naphthol Blue Black (Sigma-Aldrich $\circledast$ ).

\section{- Nucleic acid extraction and real-time RT-PCR for SARS- CoV-2 RNA detection}

The extraction of total nucleic acids (RNA and DNA) was carried out using the semiautomated NucliSENS ${ }^{\circledR}$ easyMag ${ }^{\circledR}$ platform (BioMerieux, Lyon, France) following the manufacturer's instructions. The detection of viral RNA was carried out using the AgPath-ID One-Step RT-PCR Kit (Applied Biosystems Inc., EUA) on an ABI 7500 SDS real-time PCR machine (Applied Biosystems, Weiterstadt, Germany) using a published protocol and primers and probes specific for the E [23] RNA copies/mL were quantified by real-time RT-PCR using a specific in vitro-transcribed RNA quantification standard kindly provided by Christian Drosten, Charité - Universitätsmedizin Berlin, Germany, as described previously [24].

The antiviral activity was expressed as the percent reduction in the active SARS-CoV- 2 virus RNA/mL, calculated according to Eq. (1), after contact with the test specimen compared to the number of virus particles in the positive control.

Reduction (\%) $=[(\mathrm{B}-\mathrm{A}) / \mathrm{B}] \times 100($ Eq. 1)

where $A$ and $B$ are the numbers of RNA copies $/ \mathrm{mL}$ recovered from the supernatant of APD-treated and APD-untreated cells, respectively.

\section{- Indirect Immunofluorescence (IIF)}

The methodology described here was adapted from Sales-Medina et al., 2020 [25]. Briefly, at 72 h.p.i., the plates were fixed for $30 \mathrm{~min}$ in $4 \%$ paraformaldehyde in $1 \mathrm{X}$ PBS (pH 7.4) and subjected to indirect immunofluorescence detection of viral cellular infection. After washing twice with 1X PBS $0.05 \%$ Tween 20 (PBST), the plates were blocked with bovine serum albumin (BSA) (3\% w/v in 1X PBS; Sigma-Aldrich) for 30 min at room temperature and washed twice with PBST. Convalescent serum from a Brazilian patient with COVID-19 diluted 1:1000 in PBS was used as a primary antibody to detect SARS-CoV-2 in Vero cells. The primary antibodies were incubated for $30 \mathrm{~min}$, and the plates were washed twice with PBST. Subsequently, goat anti-human IgG labeled with Alexa 488 (Thermo Scientific) diluted to $4 \mu \mathrm{g} / \mathrm{mL}$ in PBS was used as the secondary antibody, and the cells were incubated for 30 min with $5 \mu \mathrm{g} / \mathrm{mL}$ 4',6- 
diamidine-2'-phenylindole dihydrochloride (DAPI, Sigma-Aldrich) in PBS to stain the nucleic DNA. The plates were washed twice with PBST and imaged in an Operetta High Content Imaging System (Perkin Elmer) using a 20x magnification objective. Five images were acquired per well and analyzed using Harmony software (Perkin Elmer), version 3.5.2. Image analysis consisted of identifying and counting the Vero E6 cells based on the nuclear segmentation, viral infection, and cytoplasmic staining detected by the immunofluorescence assay [25].

\section{Clinical Trial Design and Oversight}

This triple-blind randomized controlled trial was conducted in accordance with the principles of the Declaration of Helsinki and ethical standards of human experimentation with the approval of the Human Research Ethics Committee of Bauru School of Dentistry of the University of Sao Paulo, Brazil (CAAE 34070620.6.0000.5417). This clinical study was also registered at REBEC - Brazilian Clinical Trial Register (RBR-58ftdj) in 10/28/2020. The study complied with the Consort 2010 checklist of information to include when reporting a randomized trial. This study was carried out as a controlled trial from 10th August to 4th November 2020 at Bauru State Hospital, Bauru, with hospitalized patients who tested positive for SARS-CoV-2. All the participants received the World Health Organization standard care hospital treatment (world medical protocol - antibiotics, corticoids and anticoagulants)[6] plus one of the two mouthwash interventions (active and nonactive mouthwashes). Based on previous studies, APD antimicrobial compound-containing mouthwash was chosen as the active mouthwash (AM) $[14,15,26]$ for comparison with a nonactive mouthwash (NAM) negative control. Both mouthwashes were produced with exactly the same formula (color, flavor, other ingredients) except for the presence or absence of the active ingredient. Once the mouthwash intervention was given to patients receiving medical treatment for COVID-19, the use of a negative control mouthwash was necessary. The active compound concentration used was between the 1:8 and 1:16 titer according to the in vitro arm of the study. All the oral care materials used during the study (mouthwashes, toothbrushes, toothpaste and dental floss) were produced by Rabbit Corp, Brazil.

\section{Patient Inclusion and Exclusion Criteria}

The inclusion criteria were patients who were 18 years old or older, who were admitted fewer than 7 days from the onset of severe acute respiratory syndrome (SARS), who were suspected of being positive for SARS-CoV-2 and who were admitted to the hospital with a mild or moderate clinical condition [6] with no need for intensive care unit (ICU). To be enrolled in the study, each participant read and signed the informed consent form after understanding the risks and objectives of the study. The exclusion criteria included patients who had contraindications to using mouthwash due to medical reasons or the inability to gargle and spit.

\section{Randomization and masking}

Sample randomization was performed as follows: the mouthwash bottles (AM and NAM) were placed in a closed package with consecutive numbers according to the patients selected. An EXCEL ${ }^{\circledR}$ database was created from these numbered packages and used for randomization. After randomization, packages 
with mouthwash bottles and oral care kits were delivered to the hospitalized patients. This study was considered triple-blind because the patients, the examiner and the statistician were blinded to the treatment groups.

\section{Mouthwash Intervention}

The eligible participants were randomly assigned to one of the following groups: AM or NAM. The patients were instructed to use $5 \mathrm{~mL}$ of the mouthwash and to switch between gargling/rinsing for $1 \mathrm{~min}$ up to 5 times a day: upon awakening, after breakfast, after lunch, after dinner and before bedtime $[14,15]$. Each patient followed this adjunctive therapeutic protocol until the outcome associated with COVID-19 medicine treatment was recorded.

\section{Outcome measures}

The primary outcome was the time to clinical improvement, defined as the length of stay in the hospital (patients did not need oxygen therapy support for more than $24 \mathrm{~h}$ and no longer showed any symptoms). Secondary outcomes were clinical evolution, need for care in the ICU and death. The criteria for the transfer of a patient to the ICU was the presence of respiratory effort requiring the use of $\mathrm{O}_{2}$ above 8.0 $\mathrm{L} / \mathrm{min}$, which is the initial graduation of nonremovable masks. The mouthwash protocol was also verified regarding the conditions of use and side effects [14, 15].

\section{Statistical analysis}

Statistical analysis was conducted using R (R Core Team) and SAS ${ }^{8}$ software version 3.8 (SAS Institute Inc). Descriptive and exploratory data analyses were performed. A Mann-Whitney U nonparametric test was used to compare the groups regarding age, number of comorbidities, and duration of symptoms prior to hospitalization. The frequency of admission to intensive care and deaths were compared between groups using Fisher's exact test. The time to clinical improvement is presented by a Kaplan-Meier plot and was compared using a Cox regression model, with an estimate of the hazard ratio association measure and 95\% confidence interval. Since a significant difference was observed between groups regarding the median age, the survival analysis was adjusted for the patient's age.

The sample of 41 patients included in this investigation provided a test power of 0.80 for a minimum detectable hazard ratio of 2.5 , with $a=0.05$, considering the follow-up time of 22 days and median hospitalization time in the control group of 7 days.

\section{Results}

\section{In vitro Cytotoxicity and Antiviral Activity}

In the optical microscopy observations, APD demonstrated cytotoxicity only at the initial dilution (2.0 $\mathrm{mg} / \mathrm{mL}$, the most concentrated stock solution) since Vero CCL-81 cell monolayer integrity was observed after treatment with all the other dilutions. The integrity of the cell monolayer was observed under a microscope after treatment with APD at dilutions ranging from $1: 2(1.0 \mathrm{mg} / \mathrm{mL})$ to $1: 32\left(6.25 \times 10^{-2}\right.$ 
$\mathrm{mg} / \mathrm{mL}$ ); these results were confirmed by cell fixation and staining with Naphthol Blue Black. The data for both the cell cytotoxicity and antiviral activity of APD were also confirmed by indirect immunofluorescence (IIF). The real-time RT-PCR results showed a significant reduction in viral load when compared to the positive control at the 1:2 (99.96\%), 1:4 (99.88\%), 1:8 (99.84\%) and 1:16 (92.65\%) titers, whereas partial virus neutralization was observed at the 1:32 (77.42\%) and 1:64 (11.06\%) titers. No virus neutralization was observed below the 1:128 titer.

\section{Clinical Trial}

\section{Patients}

According to CONSORT, 129 patients with suspected COVID-19 and admitted to the hospital were recruited for the study. After removing patients based on their oropharynx RT-PCR results, the inclusion and exclusion criteria $(n=50)$, and declining to participate or discontinuing the intervention $(n=38), 41$ patients positive for COVID-19 were finally eligible for this study. These patients were randomly divided to the AM group $(n=20)$ or the NAM group $(n=21)$ (Fig. 2$)$.

The median age of the patients was significantly higher in the AM group than in the NAM group ( $p=$ 0.0069). The participants in the NAM group were 48.4 years old on average, ranging from 27 to 70 years old, and those in the experimental group were 59.1 years old on average, ranging from 32 to 78 years old (Table 1). Additionally, $19 \%$ and $50 \%$ of the patients in the NAM and AM groups, respectively, were aged over 60 years $(p=0.0367)$. There was no significant difference between the groups regarding sex, presence of comorbidities, number of comorbidities, duration of symptoms prior to hospitalization ( $p$ > $0.05)$. 
Table 1

Demographic and clinical data of both groups of patients: active mouthwash (AM) and nonactive mouthwash (NAM) groups.

\begin{tabular}{|c|c|c|c|c|c|}
\hline & \multicolumn{4}{|l|}{ Group } & \multirow[t]{3}{*}{ p-value } \\
\hline & \multicolumn{2}{|l|}{ NAM } & \multicolumn{2}{|l|}{ AM } & \\
\hline & $\begin{array}{l}\text { Mean } \\
\text { (SD) }\end{array}$ & $\begin{array}{l}\text { Median } \\
\text { (min-max) }\end{array}$ & $\begin{array}{l}\text { mean } \\
(S D)\end{array}$ & $\begin{array}{l}\text { Median } \\
\text { (min-max) }\end{array}$ & \\
\hline Age (years) & $\begin{array}{l}48.4 \\
(11.4)\end{array}$ & $\begin{array}{l}47.0(27.0- \\
70.0)\end{array}$ & $\begin{array}{l}59.1 \\
(13.0)\end{array}$ & $\begin{array}{l}59.0(32.0- \\
78.0)\end{array}$ & ${ }^{1} 0.0069$ \\
\hline Number of comorbidities & $\begin{array}{l}1.4 \\
(1.4)\end{array}$ & $\begin{array}{l}1.0(0.0- \\
4.0)\end{array}$ & $\begin{array}{l}1.6 \\
(1.3)\end{array}$ & $\begin{array}{l}1.5(0.0- \\
5.0)\end{array}$ & ${ }^{1} 0.6481$ \\
\hline \multirow[t]{2}{*}{$\begin{array}{l}\text { Duration of symptoms prior to } \\
\text { hospitalization (days) }\end{array}$} & $\begin{array}{l}4.3 \\
(2.1)\end{array}$ & $\begin{array}{l}4.0(1.0- \\
9.0)\end{array}$ & $\begin{array}{l}5.3 \\
(1.9)\end{array}$ & $\begin{array}{l}6.0(1.0- \\
9.0)\end{array}$ & ${ }^{1} 0.1238$ \\
\hline & $\mathrm{n}$ & $\%$ & $\mathrm{n}$ & $\%$ & \\
\hline Males & 13 & $61.9 \%$ & 13 & $65.0 \%$ & ${ }^{2} 0.8370$ \\
\hline Age $>60$ y & 4 & $19.0 \%$ & 10 & $50.0 \%$ & ${ }^{2} 0.0367$ \\
\hline Presence of comorbidities & 14 & $66.7 \%$ & 16 & $80.0 \%$ & 20.3355 \\
\hline
\end{tabular}

\section{Primary outcome}

Table 2 and Fig. 3 show that there was a significant difference between the two groups regarding the occurrence of hospital discharge over time $(p<0.05)$. As there was a significant difference between the groups in terms of age, the survival analysis was performed with adjustment for age. The median LOS, that is, the time at which $50 \%$ of patients were discharged, for the NAM group was 7 days. In the AM group, this time was 4 days. It is also noted that the time for $75 \%$ of the patients in the NAM group to be discharged was 12 days, whereas in the AM group, this time was 5 days. The hazard ratio for hospital discharge was 2.16 (95\% Cl: 1.07-4.34), 
Table 2

Duration of hospitalization between groups

\begin{tabular}{|c|c|c|}
\hline & Data distribution & Time (Days) \\
\hline \multirow[t]{4}{*}{ NAM } & $75 \%(I C 95 \%)$ & $12.0(7.0-21.0)$ \\
\hline & $50 \%(I C 95 \%)$ & $7.0(4.0-10.0)$ \\
\hline & $25 \%(I C 95 \%)$ & $4.0(3.0-5.0)$ \\
\hline & Mean (SD) & $8.9(1.4)$ \\
\hline \multirow[t]{4}{*}{ AM } & $75 \%(I C 95 \%)$ & $5.0(4.0-17.0)$ \\
\hline & $50 \%(I C 95 \%)$ & $4.0(2.0-5.0)$ \\
\hline & $25 \%(I C 95 \%)$ & $2.5(1.0-3.0)$ \\
\hline & Mean (SD) & $5.10(1.19)$ \\
\hline
\end{tabular}

\section{Secondary outcomes}

There was a significant association between the need for admission to the ICU and the group to which patients belonged $(p<0.05)$. In the NAM group, $6(28.6 \%)$ patients needed intensive care during hospitalization, while in the AM group, none of the patients required ICU admission $(p=0.02)$. Three patients $(14.3 \%)$ in the NAM group and none in the experimental group died during hospitalization $(p=$ 0.23) (Table 3).

Table 3

Need for care in the Intensive Care Unit (ICU) and deaths in the NAM and AM groups.

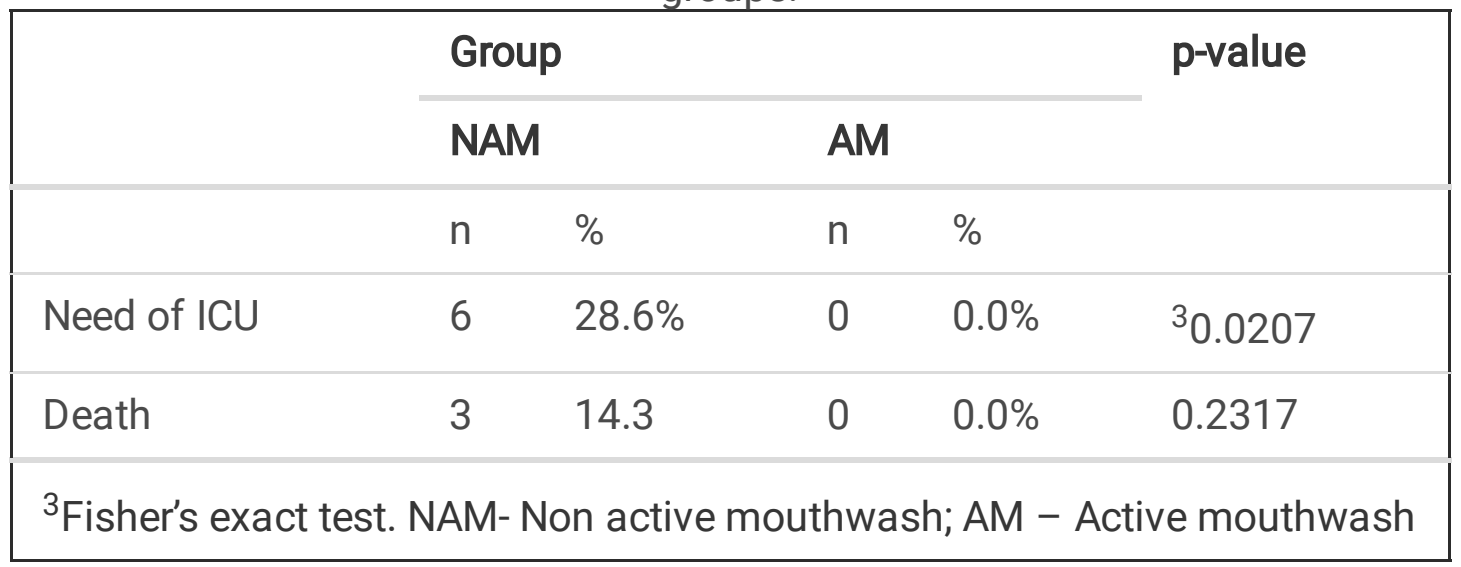

\section{Safety outcomes}


The use of the gargle/rinse protocol was possible and tolerable with no side effects. All the participants reported a pleasant, sweet taste after using both AM and NAM.

\section{Discussion}

The in vitro evaluation demonstrated promising antiviral action with the absence of cytotoxic effects in a range of concentrations of APD. The APD concentration used in the AM in this clinical study confirmed an antiviral efficacy between $92 \%$ and $99 \%$, without evidence of cytotoxicity. The AM group presented a significant reduction in the length of hospitalization and the additional benefit of less severe symptoms. Moreover, according to the safety outcomes, no side effects were reported by the patients from the AM and NAM groups regarding the gargle/rinse mouthwash protocol.

Data regarding the mean time of hospitalization due to COVID-19 vary between geographic areas, but 14 days was found to be a reliable LOS time for patients in hospitals in China [27]. In a recent study [28] was described a median hospital stay period of 12 days in Brazil, which decreased to 6 to 7 days, depending on the concentration of propolis intervention used by the patients. In the present study, a promising result was found when the median LOS further decreased to 4 days in the AM group, which differed significantly from the NAM group, which had an average LOS of 7 days. On the other hand, it is possible to hypothesize that NAM itself was effective in reducing LOS when compared to previous studies $[27,28]$. This result is probably due to mechanical hygiene of the oral and oropharynx cavities. There is in fact evidence of the effectiveness of the gargle/rinse protocol since local action is associated the regions that are intimately associated with the development of COVID-19 and play an important role in the defense of the host $[7,10,29]$. In this sense, mechanical hygiene reduces the superficial viral load of the oropharynx and oral mucosa and could prevent upper respiratory tract infections [30]. Other authors [31] reported the prevention of respiratory infections by gargling povidone-iodine, where gargling more than four times daily was considered effective in preventing the adherence of pathogenic bacteria to the upper respiratory tract. In our case, however, we believe this mechanical action was just an adjuvant that potentialized the effect of APD in reducing the SARS-CoV-2 load, which was clinically demonstrated by the great reduction in LOS. Recent studies have shown that a higher viral load is the key factor in the severity of the disease and in worse prognosis [5,32]. For this reason, decreasing the viral load is crucial to restrain the development of the disease, thus preventing the most serious symptoms. The virus enters the cell through the connection between the virus spike and the ACE2 receptor, which can be abundantly found in the salivary glands. Therefore, some recommendations have suggested the importance of controlling and reducing the viral load in the oral and oropharynx cavity by using an antiviral mouthwash $[8,9,10,11,13$, $14,33]$.

Several studies have demonstrated the antimicrobial (antiviral) and anti-inflammatory properties of phthalocyanines $[18,19,20,21]$ after photoexcitation. However, in our case, virucidal activity was observed in the dark and with an iron phthalocyanine known to have poor photochemical properties compared to the respective zinc complexes [34], indicating different mechanisms of action. 
Considering that some known technologies may exhibit an effective antagonistic action against SARSCoV-2, a virtual screening based on molecular dynamics simulations and the interaction free energies of 8.770 FDA drugs extracted from the DrugBank database (https://www.drugbank.ca/) was carried out by a team of researchers [21] which recently proposed phthalocyanine, hypericin, TMC-647055 and quarfloxin derivatives as the potentially most effective drugs for the treatment of COVID-19. All four molecules are known to have antiviral properties, but the results indicated that their high affinity for the inner cavity of the spike glycoprotein in the prefusion conformation could block the HR1 region, thus preventing the conformational changes necessary for SARS-CoV-2 entry into target cells. In other words, these molecules are potential spike glycoprotein fusion inhibitors able to prevent docking and thus infection of host cells by coronavirus.

The main interaction responsible for this fusion inhibition effect of phthalocyanine is hydrophobic, but very significant polar interactions, including hydrogen bonds, seem to be responsible for this effect of hypericin, the second most potent molecule. Accordingly, a stronger interaction and affinity of APD with the inner spike glycoprotein cavity in the prefusion conformation is expected since APD has a similar size and carboxylic acid groups at the periphery that are prone to hydrogen bond interactions. Consequently, the antiviral properties of APD could be due to its antagonistic effects on SARS-CoV-2, but APD is also known for its capacity to interact and activate oxygen molecules in the air, inducing a very localized production of activated oxygen molecules. These molecules are able to cause oxidative stress/damage to microorganisms, such as the coronavirus, leading to their inactivation. This hypothesis is confirmed by the effect of noncytotoxic, low concentrations $(1.0 \mathrm{mg} / \mathrm{mL}$ up to $0.156 \mathrm{mg} / \mathrm{mL})$ in reducing in the active viral load after proliferation in Vero CCL-81 cells as observed by RT-PCR and by the $\mathrm{HTCl}$ immunofluorescence-based assay, which showed the rapid cell nucleus localization based on DAPI staining (Fig. 1). Thus, the high effectiveness of APD can probably be explained by a dual mode of action, namely, by blocking the HR1 region and by promoting oxidative damage leading to inactivation of the virus, thus making APD a very promising molecule to reduce the SARS-CoV-2 viral load [13]. Thus, it can be suggested that a mouthwash containing APD can help improve the response of the organism to COVID-19 infection. Once the positive response of the phthalocyanine derivative-containing mouthwash in the present study and in previous studies $[14,15]$ can be observed, the authors also speculate that other properties of phthalocyanines will be identified. In this sense, the possibilities of the local effect of APD associated with the control of secondary infections (antimicrobial activity), the anti-inflammatory effect, and the modulatory effect on the immune response are not excluded $[18,19,35,36]$.

Although the COVID-19 distribution patterns in hospitals vary from one country to another [5, 6, 27], in the present study, there were no significant changes when comparing the AM and NAM groups in terms of sex, presence of comorbidities, number of comorbidities, or duration of symptoms prior to hospitalization.

The dynamics of the disease and the hospital environment, medications, stresses due to the pandemic, and sample size are some possible adverse interferences and limitations. Thus, the interpretation and generalization of the results should be performed with reservation. Nevertheless, the clinical trial clearly showed significant differences between the AM and NAM groups. In addition, the way the randomized 
study was carried out makes it unique since it created 2 nonhomogeneous groups. According to the literature [37], elderly patients who were more at risk should have taken longer to recover than younger patients. However, this was not the case, as the elderly patients in the AM group showed similar rates of recovery compared with the patients in different age groups. As a consequence, not only was the LOS decreased in this group of patients (AM) but also no intensive therapy was necessary, indicating that there was no tendency of progression into more severe prognostics. In addition, the probability of discharge (event) among participants in the AM group was twice the probability of discharge among participants in the NAM group at any point in time. Conversely, $28.6 \%$ of the patients in the NAM group needed to be admitted to the ICU, and half of this subgroup died. Further investigations are encouraged to confirm these results in larger populations.

The outstanding results achieved in the AM group suggest that mouthwash in addition to other medications can be useful in the strategic planning of COVID-19 treatment by the World Health Organization [6].

\section{Conclusion}

The APD compound was demonstrated to be highly effective in reducing the SARS-CoV-2 viral load in vitro and to exhibit no cytotoxicity in the $1.0 \mathrm{mg} / \mathrm{mL}$ to $6.25 \times 10^{-2} \mathrm{mg} / \mathrm{mL}$ range. Such a result was also confirmed in a clinical trial where gargling and rinsing five times a day was very helpful in reducing the hospital LOS for patients diagnosed with COVID-19. Further investigation is needed to elucidate this mechanism.

\section{List Of Abbreviations}

APD, Anionic phthalocyanine derivative;

WHO, World Health Organization;

AM, Active mouthwash;

NAM, Nonactive mouthwash;

LOS, Length of hospital stay;

ICU, Intensive care unit;

DMEM, Dulbecco's modified essential medium;

FBS, Fetal bovine serum;

BSA, Bovine serum albumin;

REBEC, Brazilian Clinical Trial Register; 
IIF, Indirect immunofluorescence;

ACE-2, Angiotensin-converting enzyme 2.

\section{Declarations}

\section{Author's contributions}

Manuscript concept and drafting: PSSS, BFO, FVV, KA and ELD; model development: PSSS, BFO, RRGM, FVV, LMCA, SHT, LF, KA and ELD; data collected: BFO, RRGM, LMCA, ECINP, JPGK, IAAB, DBB; data analysis: MSRZ, MQSS and ANCS; critical revision of manuscript for important intellectual content: PSSS, BFO, MSRZ, RCO, MQSS, ANCS, KA and ELD. All authors read and approved the final manuscript.

\section{Funding}

Funding This research was funded primarily by TRIALS - Oral Health \& Technologies and Golden Technology Corp. Funders contributed to the scope and design of this study; however, they did not influence the collation, management, analysis, and interpretation of the data; preparation, review, or approval of the manuscript; or the decision to submit the manuscript for publication.

This study was financed in part by the Coordenação de Aperfeiçoamento de Pessoal de Nível Superior (CAPES), Brazil (Finance Code 001).

Dr. Araki reports grants from CNPq 401581/2016-0, grants from FAPESP 18/21489-1, during the conduct of the study; in addition, Dr. Araki has a patent null pending. Dr. Da Silva Santos reports from CNPq process $n^{\circ}$. 309525/2018-7.

\section{Availability of data and materials}

The datasets generated and analysed during the current study are available from the corresponding author on reasonable request.

\section{Ethics approval and consent to participate}

This research was approved of the Human Research Ethics Committee of Bauru School of Dentistry of the University of Sao Paulo, Brazil (CAAE 34070620.6.0000.5417).

\section{Consent for publication}

Not Applicable.

\section{Competing interests}

All authors declare do not have any competing interests. 


\section{References}

1. Zhou, F. et al. Clinical course and risk factors for mortality of adult inpatients with COVID-19 in Wuhan, China: a retrospective cohort study. Lancet. 395, 10229 (2020).

2. Lu, R. et al. Genomic characterisation and epidemiology of 2019 novel coronavirus: implications for virus origins and receptor binding. Lancet. 395, 10224 (2020).

3. Zhu, N. et al. China Novel Coronavirus Investigating and Research Team. A Novel Coronavirus from Patients with Pneumonia in China, 2019. N Engl J Med. 382, 8 (2020).

4. https://. Accessed 30 Jan 2021.

5. Magleby, R. et al. Impact of SARS-CoV-2 Viral Load on Risk of Intubation and Mortality Among Hospitalized Patients with Coronavirus Disease 2019.Clin Infect Dis. 2020;ciaa851.

6. https://. Accessed: 30 Jan 2021.

7. Tashiro, A. \& Shaw, R. COVID-19 Pandemic Response in Japan: What Is behind the Initial Flattening of the Curve? Sustainability. 12, 5250 (2020).

8. O'Donnell, V. B. et al. Potential Role of Oral Rinses Targeting the Viral Lipid Envelope in SARS-CoV-2 Infection. Function (Oxf). 1, 1 (2020).

9. Xu, J., Li, Y., Gan, F., Du, Y. \& Yao, Y. Salivary Glands: Potential Reservoirs for COVID-19 Asymptomatic Infection. J Dent Res. 99, 8 (2020).

10. Naqvi, S. H. S. et al. Povidone-iodine solution as SARS-CoV-2 prophylaxis for procedures of the upper aerodigestive tract a theoretical framework. J Otolaryngol Head Neck Surg. 49, 1 (2020).

11. Carrouel, F. et al. COVID-19: A Recommendation to Examine the Effect of Mouthrinses with $\beta$ Cyclodextrin Combined with Citrox in Preventing Infection and Progression. J Clin Med. 9, 4 (2020).

12. Meister, T. L. et al. Virucidal Efficacy of Different Oral Rinses Against Severe Acute Respiratory Syndrome Coronavirus 2. J Infect Dis. 222, 8 (2020).

13. Santos, P. S. S. et al. A Recommendation of PHTALOX® Mouthwash for Preventing Infection and Progression of COVID-19. Acta Scient Dent Sci. 4, 12 (2020).

14. da Fonseca Orcina, B. et al. A Phthalocyanine Derivate Mouthwash to Gargling/Rinsing as an Option to Reduce Clinical Symptoms of COVID-19: Case Series. Clin Cosmet Investig Dent. 13, 47-50 (2021).

15. Orcina, B. F. \& Santos, P. S. S. Oral Manifestation COVID-19 and the Rapid Resolution of Symptoms Post-Phtalox Treatment: a Case Series. Int J. Odontostomat. 15 (1), 67-70 (2021).

16. https://vihema.gov.vn. Accessed 30 Jan 2021.

17. https://. Accessed 30 Jan 2021.

18. Moussaron, A. et al. Lipophilic phthalocyanines for their potential interest in photodynamic therapy: synthesis and photo-physical properties. Tetrahedron. 69 (47), 10116-10122 (2013).

19. Heugebaert, T. S., Roman, B. I. \& Stevens, C. V. Synthesis of isoindoles and related iso-condensed heteroaromatic pyrroles. Chem Soc Rev. 41 (17), 5626-5640 (2012). 
20. Encinar, J. A. \& Menendez, J. A. Potential Drugs Targeting Early Innate Immune Evasion of SARSCoronavirus 2 via 2'-O-Methylation of Viral RNA. Viruses. 12 (5), 525 (2020).

21. Romeo, A., lacovelli, F. \& Falconi, M. Targeting the SARS-CoV-2 spike glycoprotein prefusion conformation: virtual screening and molecular dynamics simulations applied to the identification of potential fusion inhibitors. Virus Res. 286, 198068 (2020).

22. Araujo, D. B. et al. SARS-CoV-2 isolation from the first reported patients in Brazil and establishment of a coordinated task network. Mem Inst Oswaldo Cruz. 115, e200342 (2020).

23. Corman, V. M. et al. Detection of 2019 novel coronavirus (2019-nCoV) by real-time RT-PCR. Euro Surveill. 25 (3), 200045 (2020).

24. Drosten, C. et al. Identification of a novel coronavirus in patients with severe acute respiratory syndrome. N Engl J Med. 348 (20), 1967-1976 (2003).

25. Sales-Medina, D. F. et al. Discovery of clinically approved drugs capable of inhibiting SARS-CoV-2 in vitro infection using a phenotypic screening strategy and network-analysis to predict their potential to treat covid-19. bioRxiv. 2020. https://doi.org/10.1101/2020.07.09.196337.

26. Teodoro, G. R. et al. PHTALOX® antimicrobial action and cytotoxicity: in vitro study. J Dent Res 99(Speclss A): abstract number, 0839, 2020 IADR/AADR/CADR General Session (Washington, D.C., USA).

27. Rees, E. M. et al. CA, et al. COVID-19 length of hospital stay: a systematic review and data synthesis. BMC Med. 18 (1), 270 (2020).

28. Silveira, M. A. D. et al. Efficacy of propolis as an adjunct treatment for hospitalized COVID-19 patients: a randomized, controlled clinical trial. MedRxiv.

https://doi.org/10.1101/2021.01.08.20248932 (2021).

29. Hakansson, A. P., Orihuela, C. J. \& Bogaert, D. Bacterial-Host Interactions: Physiology and Pathophysiology of Respiratory Infection. Physiol Rev. 98 (2), 781-811 (2018).

30. Satomura, K. et al. Prevention of upper respiratory tract infections by gargling: a randomized trial. Am J Prev Med. 29 (4), 302-307 (2005).

31. Nagatake, T., Ahmed, K. \& Oishi, K. Prevention of respiratory infections by povidone-iodine gargle. Dermatology. 204 (Suppl 1), 32-36 (2002).

32. Liu, Y. et al. Viral dynamics in mild and severe cases of COVID-19. Lancet Infect Dis. 20 (6), 656-657 (2020).

33. de Telles-Araujo, T., Caminha, G., Kallás, R. D. G., Sipahi, M. S. \& da Silva Santos, A. M. PS. Potential mouth rinses and nasal sprays that reduce SARS-CoV-2 viral load: What we know so far? Clinics (Sao Paulo). 75, e2328 (2020).

34. Kawauchi, K. et al. An anionic phthalocyanine decreases NRAS expression by breaking down its RNA G-quadruplex. Nat Commun. 9 (1), 2271 (2018).

35. Sun, S., Jiang, N. \& Xia, D. Density Functional Theory Study of the Oxygen Reduction Reaction on Metalloporphyrins and Metallophthalocyanines. J Phys Chem. 115 (19), 9511-9517 (2011). 
36. Gadotti, A. C. et al. Susceptibility of the patients infected with Sars-Cov2 to oxidative stress and possible interplay with severity of the disease. Free Radic Biol Med. 165, 184-190 (2021).

37. Thai, P. Q. et al. Factors associated with the duration of hospitalisation among COVID-19 patients in Vietnam: A survival analysis. Epidemiol Infect. 148, e114 (2020).

\section{Figures}

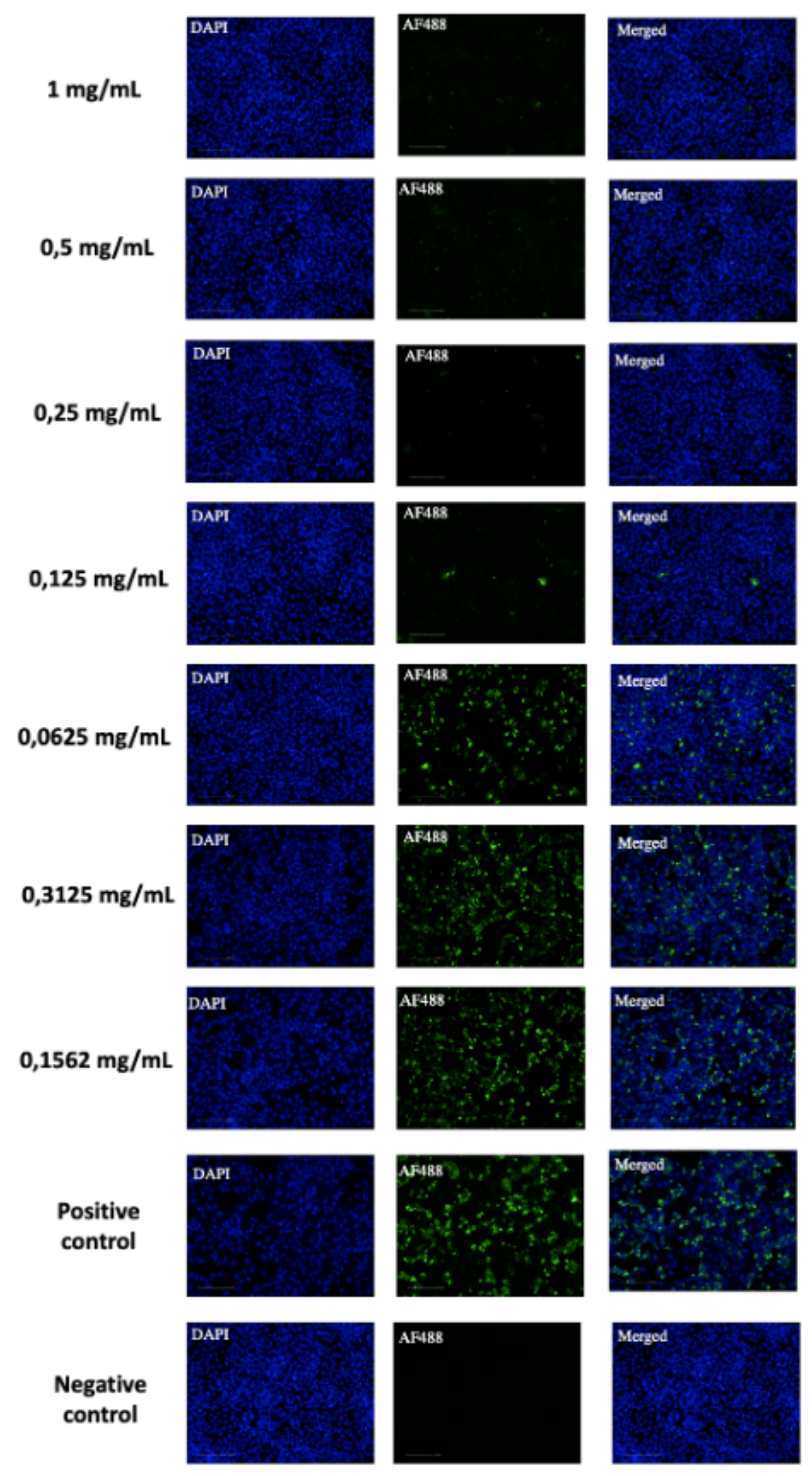




\section{Figure 1}

Indirect immunofluorescence (IIF) assay for the detection of SARS-CoV-2-infected cells. Representative images of the noncytotoxic concentrations $(1.0 \mathrm{mg} / \mathrm{mL}$ up to $0.156 \mathrm{mg} / \mathrm{mL})$ of APD observed with a $20 \mathrm{x}$ objective. A mixture of MERS-CoV-infected and noninfected Vero cells was stained with convalescent serum monoclonal antibodies, followed by incubation with the Alexa488-conjugated goat anti-human IgG antibodies (green). Cells were counterstained with DAPI to stain the nuclei (blue). Positive (infected nontreated cells) and negative (noninfected cells) controls are shown at the bottom of the image. Images were taken using the Operetta High Content Imaging System (Perkin Elmer). Scale bar, $100 \mu \mathrm{m}$. 


\section{$\Rightarrow$ CONSORT \\ TRANSPARENT REPORTING of TRIALS}

\section{CONSORT 2010 Flow Diagram}

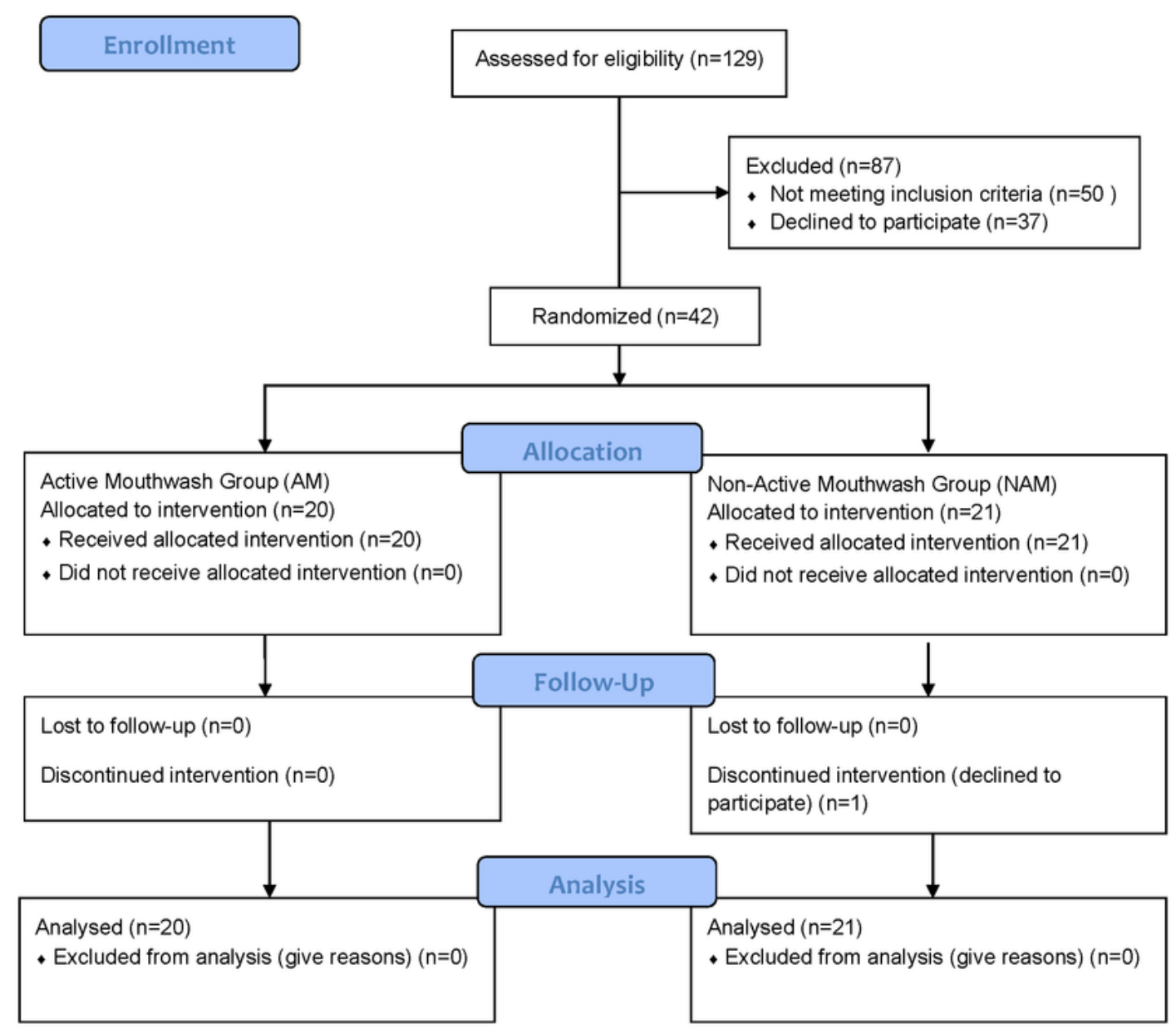

Figure 2

Consort Flow Diagram 


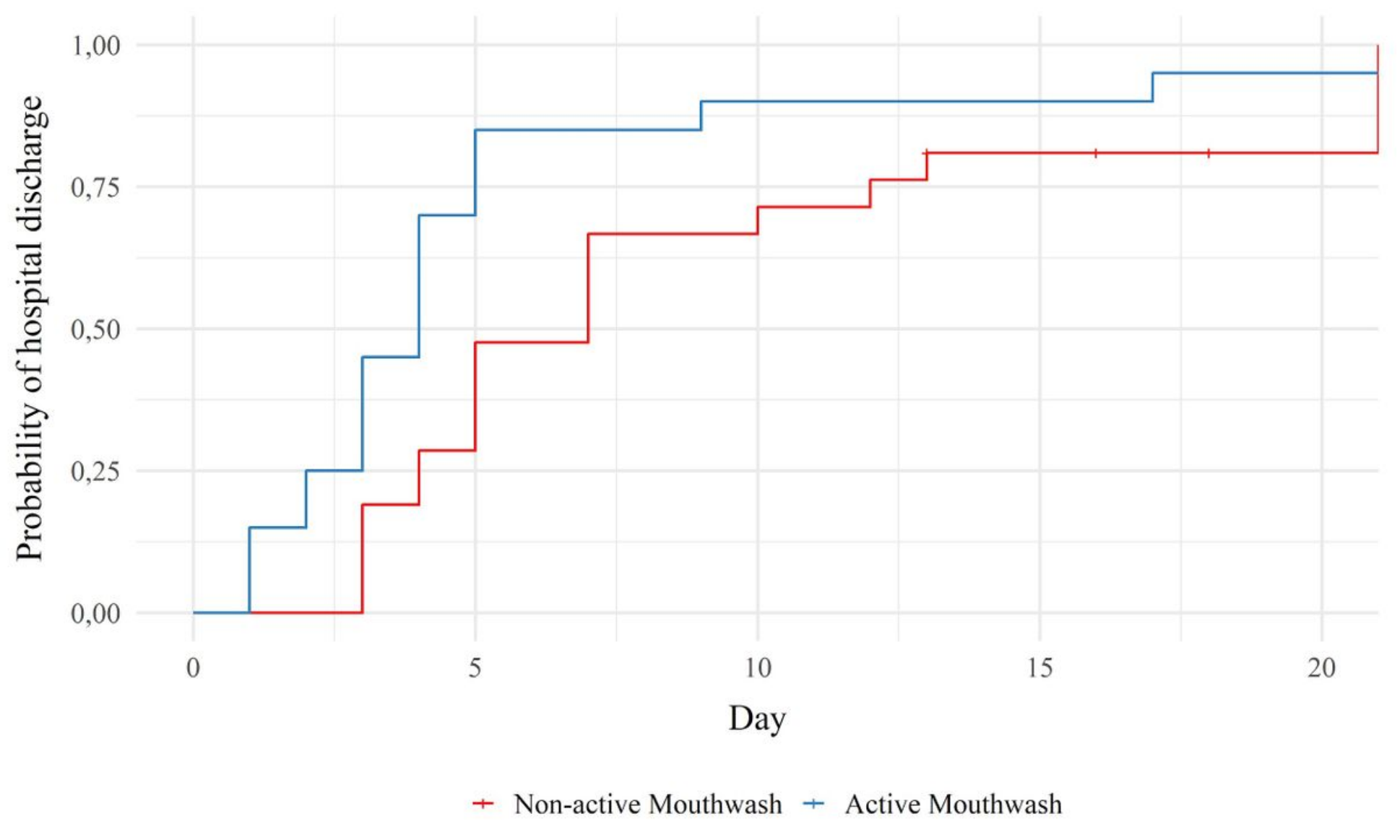

Figure 3

Probability of hospital discharge over time (days) according to each group.

\section{Supplementary Files}

This is a list of supplementary files associated with this preprint. Click to download.

- Supplementarylnformation.docx 